\title{
A Balancing Algorithm for Mortar Methods
}

\author{
Dan Stefanica \\ Baruch College, City University of New York, NY 10010, USA \\ Dan_Stefanica@baruch.cuny.edu
}

Summary. The balancing methods are hybrid nonoverlapping Schwarz domain decomposition methods from the Neumann-Neumann family. They are efficient and easy to implement. We present a new balancing algorithm for mortar finite element methods. We prove a condition number estimate which depends polylogarithmically on the number of nodes on each subregion edge and does not depend on the number of subregions from the partition of the computational domain, just as in the continuous case.

\section{Introduction}

The balancing method of Mandel [7] is a hybrid nonoverlapping Schwarz domain decomposition method from the Neumann-Neumann family. It is easy to implement and uses a natural coarse space of minimal dimension which allows for an unstructured partition of the computational domain. The condition numbers of the resulting algorithms depend polylogarithmically on the number of degrees of freedom in each subregion. There is a close connection between the balancing method and the FETI [5] and FETI-DP [4]; cf. [6]. A new version of the balancing method, also related to FETI-type algorithms, was recently proposed by Dohrmann et al. [9, 10].

Mortar finite elements were first introduced by Bernardi et al. [2] and are actively used in practice for their advantages over the conforming finite elements, e.g., flexible mesh generation and straightforward local refinement. In this paper, we propose an extension of the balancing method to mortar finite elements. As in the continuous case, every local space is associated with a subregion from the partition of the computational domain. The values of the mortar function on a nonmortar side depend on, but are not equal to, its values on the mortar sides opposite the nonmortar. To account for this dependence, the local are spaces defined on extended subregions, instead of using local spaces and local solvers defined on each subregion. In this regard, our algorithm is different from classical Neumann-Neumann methods. 
We establish a polylogarithmic upper bound for the condition number of our algorithm. The same bound was obtained for the balancing algorithm of Dryja [3], as well as for other mortar algorithms, e.g., the iterative substructuring method of Achdou et al. [1], in the geometrically nonconforming case.

While the algorithm proposed here is based on a similar philosophy to the method suggested in [3], since the Schwarz framework is used to study the convergence properties of both algorithms, major differences exist between the two algorithms. For example, in the algorithm [3], the local spaces are associated with pairs of opposite nonmortar and mortar sides.

\section{Abstract Schwarz Theory}

We use this elegant framework of the abstract Schwarz theory [11] to study the convergence properties of the balancing algorithm proposed in this paper.

Let $V$ be a finite dimensional space, with a coercive inner product $a$ : $V \times V \rightarrow \mathrm{R}$, and let $f: V \rightarrow \mathrm{R}$ be a continuous operator. We want to find the unique solution $u \in V$ of

$$
a(u, v)=f(v), \quad \forall v \in V .
$$

Assume that $V$ can be written as $V=V_{0}+V_{1}+\ldots+V_{N}$, where the sum is not necessarily direct and $V_{i} \subset V, i=0: N$. Let $I_{i}: V_{i} \rightarrow V$ be embedding operators and let $\tilde{a}_{i}: V_{i} \times V_{i} \rightarrow \mathrm{R}$ be bilinear forms which are symmetric, continuous, and coercive. The corresponding projection-like operators $\widetilde{T}_{i}: V \rightarrow V_{i}$ are defined by

$$
\tilde{a}_{i}\left(\widetilde{T}_{i} v, v_{i}\right)=a\left(v, I_{i} v_{i}\right), \quad \forall v_{i} \in V_{i}, v \in V .
$$

Using the operators $T_{i}: V \rightarrow V, T_{i}=I_{i} \widetilde{T}_{i}$, the additive and multiplicative Schwarz methods for solving (1) can be introduced.

The balancing method is a hybrid method, combining the potential for parallelization of the additive methods and the fast convergence of the multiplicative methods. Choose the bilinear form $\tilde{a}_{0}$ to be exact, i.e., $\tilde{a}_{0}(\cdot, \cdot)=a(\cdot, \cdot)$. The coarse space solver $T_{0}$ is therefore a projection, subsequently denoted by $P_{0}$. The balancing method consists of solving $T_{b a l} u=g_{b a l}$, where

$$
T_{b a l}=P_{0}+\left(I-P_{0}\right)\left(T_{1}+\ldots+T_{N}\right)\left(I-P_{0}\right) .
$$

Here, $g_{b a l}$ is obtained by solving $N$ local problems of the same form as (2) that do not require any knowledge of $u$. The equation $T_{b a l} u=g_{b a l}$ is a preconditioned version of (1) and can be solved without further preconditioning using CG or GMRES algorithms. 


\section{A Mortar Discretization of an Elliptic Problem}

As model problem for two dimensional self-adjoint elliptic PDEs with homogeneous coefficients, we choose the Poisson problem with mixed boundary conditions on $\Omega$ : Given $f \in L^{2}(\Omega)$, find $u \in H^{1}(\Omega)$ such that

$$
-\Delta u=f \text { on } \Omega, \quad \text { with } u=0 \text { on } \partial \Omega_{D} \text { and } \partial u / \partial n=0 \text { on } \partial \Omega_{N},
$$

where $\partial \Omega_{N}$ and $\partial \Omega_{D}$ are the parts of $\partial \Omega=\partial \Omega_{N} \cup \partial \Omega_{D}$ where Neumann and Dirichlet boundary conditions are imposed, respectively, and $\partial \Omega_{D}$ has positive Lebesgue measure.

To keep the presentation concise, we only discuss geometrically conforming mortar elements. Let $\left\{\Omega_{i}\right\}_{i=1: N}$ be a geometrically conforming mortar decomposition of a polygonal domain $\Omega$ of diameter 1 into rectangles of diameter of order $H$. (This notation is not coincidental: for the balancing method proposed here, each local space $V_{i}$ will correspond to one subregion $\Omega_{i}$.) The restriction of the mortar finite element space $V^{h}$ to any rectangle $\Omega_{i}$ is a $Q_{1}$ finite element function on a mesh of diameter $h$. Weak continuity is required across $\Gamma$, the interface between the subregions $\left\{\Omega_{i}\right\}_{i=1: N}$. We choose a set of edges of $\left\{\Omega_{i}\right\}_{i=1: N}$, called nonmortars, which form a disjoint partition of $\Gamma$. For each nonmortar side $\gamma$ there exists exactly one side opposite to it, which is called a mortar side. The jump $[w]$ of a mortar function $w \in V$ across any nonmortar $\gamma$ must be orthogonal to a space of test functions $\Psi(\gamma)$, i.e.,

$$
\int_{\gamma}[w] \psi d s=0, \quad \forall \psi \in \Psi(\gamma) .
$$

In [2], $\Psi(\gamma)$ consists of continuous, piecewise linear functions on $\gamma$ that are constant in the first and last mesh intervals of $\gamma$. Note that the end points of the nonmortar sides are associated with genuine degrees of freedom.

We discretize the Poisson problem (4) by using the mortar finite element space $V^{h}$. and obtain the discrete problem

$$
\text { Find } u_{h} \in V^{h} \text { such that } a^{\Gamma}\left(u_{h}, v_{h}\right)=f\left(v_{h}\right), \quad \forall v_{h} \in V^{h} \text {, }
$$

where the bilinear form $a^{\Gamma}(\cdot, \cdot)$ is defined as the sum of contributions from the individual subregions, and $f(\cdot)$ is the $L^{2}$-inner product by the function $f$ :

$$
a^{\Gamma}\left(v_{h}, w_{h}\right)=\sum_{i=1}^{N} \int_{\Omega_{i}} \nabla v_{h} \cdot \nabla w_{h} d x \text { and } f(v)=\int_{\Omega} f v d x
$$

Let $V^{h}(\Gamma)$ be the restriction of $V^{h}$ to the interface $\Gamma$, and let $V$ be the space of discrete piecewise harmonic functions defined as follows: If $v_{\Gamma} \in$ $V^{h}(\Gamma)$, then its harmonic extension $\mathcal{H}\left(v_{\Gamma}\right) \in V$ is the only function in $V^{h}$ which, on every subregion $\Omega_{i}$, is equal to the harmonic extension of $\left.v_{\Gamma}\right|_{\partial \Omega_{i}}$ with respect to the $H^{1}$-seminorm. 
As in other substructuring methods, we eliminate the unknowns corresponding to the interior of the subregions. Problem (6) becomes a Schur complement problem on $V^{h}(\Gamma)$ :

Find $u_{\Gamma} \in V^{h}(\Gamma) \quad$ s.t. $\quad a^{\Gamma}\left(\mathcal{H}\left(u_{\Gamma}\right), \mathcal{H}\left(v_{\Gamma}\right)\right)=f\left(\mathcal{H}\left(v_{\Gamma}\right)\right), \forall v_{\Gamma} \in V^{h}(\Gamma)$.

For simplicity, we denote $V^{h}(\Gamma)$ by $V$ and let $a(\cdot, \cdot)=a^{\Gamma}(\mathcal{H}(\cdot), \mathcal{H}(\cdot))$ be the inner product on $V$. Problem (7) can be formulated on $V$ as follows:

$$
\text { Find } u \in V \text { s.t. } a(u, v)=f(v), \quad \forall v \in V \text {. }
$$

\section{A Balancing Algorithm for Mortars}

In this section, we introduce a new balancing algorithm for mortar finite elements. Our results can be extended to second order self-adjoint elliptic problems with mixed boundary conditions discretized by geometrically nonconforming mortars, and to three dimensional problems.

We solve (8) using the technique outlined in Section 2. To do so, we need to introduce a coarse space $V_{0}$ and local spaces $V_{i}, i=1: N$. The major difference between the classical balancing method and our algorithm for mortars is related to the extended subregions $\widetilde{\Omega}_{i}$, which replace the individual subregions in the definition of the local bilinear forms $\tilde{a}_{i}(\cdot, \cdot)$. An important role in the balancing algorithm is played by the counting functions associated with the interface nodes of each extended subregion. In [12], we showed that defining $\tilde{a}_{i}(\cdot, \cdot)$ only on $\Omega_{i}$ does not lead to a convergent algorithm.

Extended Subregions: The extended subregion $\widetilde{\Omega}_{i}$ is defined as the union of $\Omega_{i}$ and all its neighbors that have a mortar side opposite $\partial \Omega_{i}$. Let $\mathrm{N}_{i}$ be the set made of the corner nodes of $\Omega_{i}$, all the nodes on the mortar sides of $\Omega_{i}$, and all the nodes on the mortar sides opposite the nonmortar sides of $\Omega_{i}$.

The counting function $\nu_{i}: \Gamma \rightarrow \mathrm{R}$ corresponding to $\Omega_{i}$ is a mortar function taking the following values at the genuine degrees of freedom:

$$
\nu_{i}(x)=\left\{\begin{array}{cl}
\text { number of sets } \mathrm{N}_{j} \text { with } x \in \mathrm{N}_{j}, & \text { if } x \in \mathrm{N}_{i} ; \\
0, & \text { if } x \notin \mathrm{N}_{i} ; \\
1, & \text { if } x \in \partial \Omega_{i} \cap \partial \Omega_{D} .
\end{array}\right.
$$

In the geometrically conforming case, the value of $\nu_{i}$ at every interior node of the mortar sides where $\nu_{i}$ does not vanish is equal to 2 , and Range $\left(\nu_{i}\right) \subseteq$ $\{0,1, \ldots, 4\}$. Let $\nu_{i}^{\dagger}$ be the mortar function with nodal values $\nu_{i}^{\dagger}(x)=1 / \nu_{i}(x)$ if $\nu_{i}(x) \neq 0$ and $\nu_{i}^{\dagger}(x)=0$ otherwise. As in the continuous finite element case, $\nu_{i}^{\dagger}$ form a partition of unity, i.e., $\sum_{i=1}^{N} \nu_{i}^{\dagger}=1$.

Coarse Space $V_{0}$ : The coarse space $V_{0}$ has one basis function, $\mathcal{H}\left(\nu_{i}^{\dagger}\right)$, the harmonic extension of $\nu_{i}^{\dagger}$, per subregion $\Omega_{i}$. The bilinear form $a_{0}$ is exact, i.e., $\tilde{a}_{0}(\cdot, \cdot)=a(\cdot, \cdot)$. Therefore, $a\left(P_{0} u, \mathcal{H}\left(\nu_{i}^{\dagger}\right)\right)=a\left(u, \mathcal{H}\left(\nu_{i}^{\dagger}\right)\right)$, and 

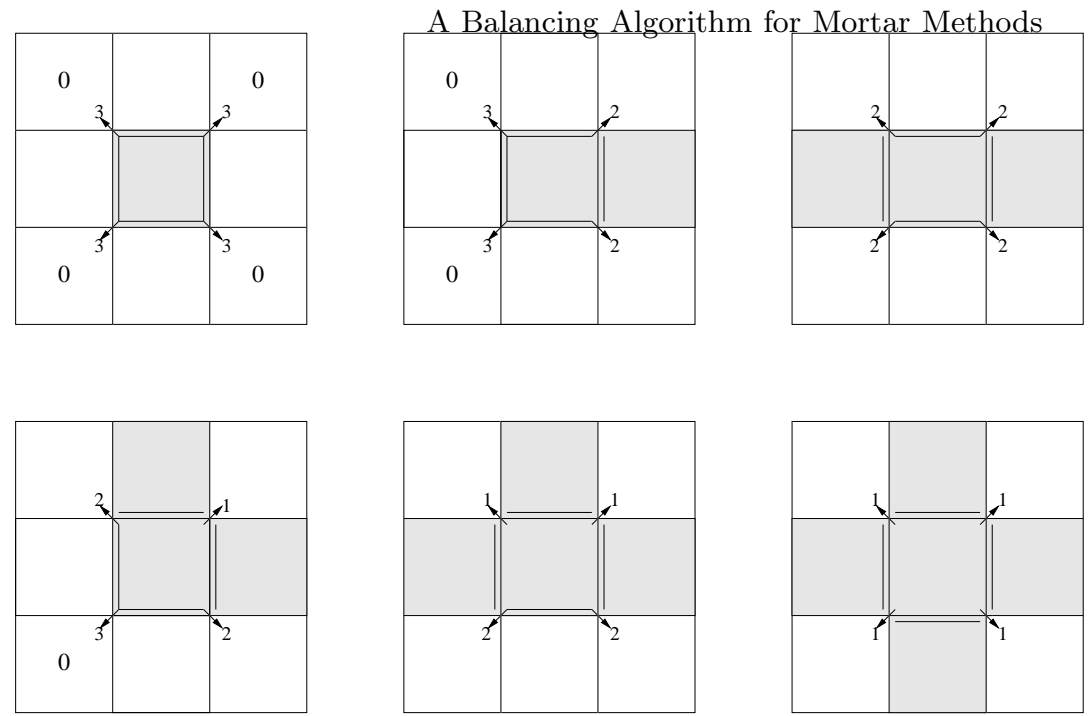

Fig. 1. All possible instances of extended subregions $\widetilde{\Omega}_{i}$ (shaded) corresponding to one subregion $\Omega_{i}$ (center in each picture). The values of the counting function $\nu_{i}$ at the corners of $\Omega_{i}$ are recorded. Mortar sides are marked with an additional solid line.

$$
a\left(\left(I-P_{0}\right) u, \mathcal{H}\left(\nu_{i}^{\dagger}\right)\right)=0, \quad \forall u \in V
$$

Local Spaces: The local space $V_{i}$ is associated with the subregion $\Omega_{i}$, is embedded in $V$, i.e., $V_{i} \subset V$, and consists of piecewise harmonic functions which vanish at all the genuine degrees of freedom of $\Gamma \backslash \mathrm{N}_{i}$. The bilinear form $\tilde{a}_{i}(\cdot, \cdot): V_{i} \times V_{i} \rightarrow R$ is defined using the extended subregion $\widetilde{\Omega}_{i}$ :

$$
\tilde{a}_{i}\left(v_{i}, w_{i}\right)=\sum_{\Omega_{j} \subset \widetilde{\Omega}_{i}} \int_{\Omega_{j}} \nabla \mathcal{H}\left(I_{h}\left(\nu_{i} v_{i}\right)\right) \cdot \nabla \mathcal{H}\left(I_{h}\left(\nu_{i} w_{i}\right)\right) d x
$$

where $I_{h}: L^{2}(\Omega) \rightarrow V$ is the nodal basis interpolation onto the mortar space $V$. The projection-like operator $T_{i}$ is given by $T_{i}=I_{i} \widetilde{T}_{i}$, where

$$
\tilde{a}_{i}\left(\widetilde{T}_{i} u, v_{i}\right)=a\left(u, v_{i}\right), \quad \forall v_{i} \in V_{i}
$$

If $\widetilde{\Omega}_{i} \neq \Omega_{i}$, i.e., if $\widetilde{\Omega}_{i}$ contains more than one subregion, then any $v_{i} \in V_{i}$ vanishes on $\partial \widetilde{\Omega}_{i} \backslash \partial \Omega_{i}$. The problem (11) is well-posed since it is a Poisson problem on $\widetilde{\Omega}_{i}$ with zero Dirichlet boundary conditions on $\partial \widetilde{\Omega}_{i} \backslash \partial \Omega_{i}$.

If $\widetilde{\Omega}_{i}=\Omega_{i}$, then all the sides of $\Omega_{i}$ are mortars; cf. Figure 1, upper left picture. This corresponds to the case of a floating subregion in the classical balancing algorithm, and requires using balanced functions. Note that $\mathcal{H}\left(\nu_{i} \nu_{i}^{\dagger}\right)$ is equal to 1 on $\Omega_{i}$, and therefore 


$$
\tilde{a}_{i}\left(\widetilde{T}_{i} u, \mathcal{H}\left(\nu_{i}^{\dagger}\right)\right)=\int_{\Omega_{i}} \nabla \mathcal{H}\left(I_{h}\left(\nu_{i} \widetilde{T}_{i} u\right)\right) \cdot \nabla \mathcal{H}\left(\nu_{i} \nu_{i}^{\dagger}\right) d x=0 .
$$

For the local problem (11) to be solvable, $u$ must satisfy

$$
a\left(u, \mathcal{H}\left(\nu_{i}^{\dagger}\right)\right)=0
$$

for every floating subregion $\Omega_{i}$. Such functions are called balanced functions. From (9), we conclude that any function in $\operatorname{Range}\left(I-P_{0}\right)$ is balanced.

Moreover, if $\widetilde{\Omega}_{i}=\Omega_{i}$, the local problem (11) corresponds to a pure Neumann problem. We make the solution unique by requiring $\widetilde{T}_{i} u$ to satisfy

$$
\int_{\Omega_{i}} \mathcal{H}\left(I_{h}\left(\nu_{i} \widetilde{T}_{i} u\right)\right) d x=0 .
$$

The preconditioned operator for our balancing algorithm for mortars is $T_{b a l}=P_{0}+\left(I-P_{0}\right)\left(T_{1}+\ldots+T_{N}\right)\left(I-P_{0}\right)$. The convergence analysis of $T_{b a l}$ relies on that of the Neumann-Neumann operator $T_{N-N}=P_{0}+T_{1}+$ $\ldots+T_{N}$, since $\kappa\left(T_{\text {bal }}\right) \leq \kappa\left(T_{N-N}\right)$; cf., e.g., [8]. However, Neumann-Neumann algorithms with the spaces and approximate solvers considered in this paper would not converge.

\section{Condition number estimate}

The condition number estimate for our algorithm is based on abstract Schwarz theory; see, e.g., [11]. A technical results has to be proven first, and the techniques are somewhat different for floating and non-floating regions:

Lemma 1. Let $u \in V$ and let $\bar{u}_{i}=\mathcal{H}\left(I_{h}\left(\nu_{i}^{\dagger}\left(u-\alpha_{i}\right)\right)\right) \in V_{i}$, where $\alpha_{i}$ is the weighted averages of $u$ over $\Omega_{i}$, i.e.,

$$
\alpha_{i}=\frac{1}{\mu\left(\Omega_{i}\right)} \int_{\Omega_{i}} u d x
$$

Then

$$
\begin{aligned}
& a\left(u_{i}, u_{i}\right) \leq C(1+\log (H / h))^{2} \tilde{a}_{i}\left(u_{i}, u_{i}\right), \quad \forall u_{i} \in \operatorname{Range}\left(T_{i}\right) \\
& a\left(\bar{u}_{i}, \bar{u}_{i}\right) \leq C(1+\log (H / h))^{2}|u|_{H^{1}\left(\widetilde{\Omega}_{i}\right)}^{2} .
\end{aligned}
$$

Also, if $\Omega_{i}$ is a floating subregion, i.e., if $\widetilde{\Omega}_{i}=\Omega_{i}$, then $\tilde{a}_{i}\left(\bar{u}_{i}, \bar{u}_{i}\right)=|u|_{H^{1}\left(\widetilde{\Omega}_{i}\right)}^{2}$. If $\Omega_{i}$ is a nonfloating subregion, i.e., if $\widetilde{\Omega}_{i} \neq \Omega_{i}$, then $\tilde{a}_{i}\left(\bar{u}_{i}, \bar{u}_{i}\right) \leq C(1+$ $\log (H / h))^{2}|u|_{H^{1}\left(\widetilde{\Omega}_{i}\right)}^{2}$ 
Using the results of Lemma 1, we can show that $\tilde{a}_{i}(\cdot, \cdot)$ is bounded from below by $a(\cdot, \cdot)$, and prove that for any function in $V$ there exists a stable splitting into local functions; see [12] for detailed proofs.

Lemma 2. There exists a constant $C$, not depending on the local spaces $V_{i}$, such that

$$
a\left(u_{i}, u_{i}\right) \leq C(1+\log (H / h))^{2} \tilde{a}_{i}\left(u_{i}, u_{i}\right), \quad \forall u_{i} \in \operatorname{Range}\left(T_{i}\right), \quad \forall i=1: N .
$$

Lemma 3. Let $u \in V$ and let $\alpha_{i}$ be the weighted averages 14 of $u$ over $\Omega_{i}$. Define $u_{0} \in V_{0}$ as $u_{0}=\sum_{i=1}^{N} \alpha_{i} \mathcal{H}\left(\nu_{i}^{\dagger}\right)$ and let $\bar{u}_{i} \in V_{i}$ be given by $\bar{u}_{i}=$ $\mathcal{H}\left(I_{h}\left(\nu_{i}^{\dagger}\left(u-\alpha_{i}\right)\right)\right)$. Then $u=u_{0}+\sum_{i=1}^{N} \bar{u}_{i}$ and

$$
a\left(u_{0}, u_{0}\right)+\sum_{i=1}^{N} \tilde{a}_{i}\left(\bar{u}_{i}, \bar{u}_{i}\right) \leq C(1+\log (H / h))^{2} a(u, u) .
$$

Based on the results of Lemmas 2 and 3, a bound on $\kappa\left(T_{N-N}\right)$, and therefore on $\kappa\left(T_{b a l}\right)$, can be established by using the abstract Schwarz theory [11].

Theorem 1. The condition number of the balancing algorithm is independent of the number of subregions and grows at most polylogarithmically with the number of nodes in each subregion, i.e.,

$$
\kappa\left(T_{b a l}\right) \leq C(1+\log (H / h))^{4},
$$

where $C$ is a constant that does not depend on the properties of the partition.

\section{Numerical Results}

We tested the convergence properties of our balancing algorithm for a two dimensional problem discretized by geometrically nonconforming mortar finite elements. The model problem was the Poisson equation on the unit square $\Omega$ with mixed boundary conditions. We partitioned $\Omega$ into $N=16,32,64$, and 128 geometrically nonconforming rectangles, and $Q_{1}$ elements were used in each square. For each partition, the number of nodes on each edge, $H / h$, was taken to be, on average, $4,8,16$, and 32 , respectively, for different sets of experiments. The preconditioned conjugate gradient iteration was stopped when the residual norm decreased by a factor of $10^{-6}$. The experiments were carried out in MATLAB. We report iteration counts, condition number estimates, and flop counts of our algorithm in Table 1.

Our balancing algorithm has similar scalability properties as those of the classical balancing algorithm. When the number of nodes on each subregion edge, $H / h$, was fixed and the number of subregions, $N$, was increased, the iteration count showed only a slight growth. When $H / h$ was increased, while the partition was kept unchanged, the small increase in the number of iterations was satisfactory. The condition number estimates exhibited a similar dependence, or lack thereof, on $N$ and $H / h$. 
Table 1. Convergence results, geometrically nonconforming mortars

\begin{tabular}{|l|r|rrr||r||r|r|rr|}
\hline$N$ & $H / h$ & Iter Cond Mflops & \multicolumn{3}{|c}{$H / h$} & Iter Cond Mflops \\
\hline \hline 16 & 4 & 11 & 9.2 & $4.7 \mathrm{e}-1$ & 64 & 4 & 14 & 9.9 & $4.0 \mathrm{e}+0$ \\
16 & 8 & 13 & 10.8 & $2.6 \mathrm{e}+0$ & 64 & 8 & 15 & 12.1 & $1.6 \mathrm{e}+1$ \\
16 & 16 & 14 & 12.1 & $1.6 \mathrm{e}+1$ & 64 & 16 & 17 & 13.4 & $9.4 \mathrm{e}+1$ \\
16 & 32 & 15 & 13.3 & $1.3 \mathrm{e}+2$ & 64 & 32 & 19 & 13.9 & $7.2 \mathrm{e}+2$ \\
\hline 32 & 4 & 12 & 9.6 & $1.5 \mathrm{e}+0$ & 128 & 4 & 14 & 10.3 & $1.0 \mathrm{e}+1$ \\
32 & 8 & 14 & 11.3 & $7.2 \mathrm{e}+0$ & 128 & 8 & 15 & 12.0 & $3.6 \mathrm{e}+1$ \\
32 & 16 & 15 & 12.9 & $4.5 \mathrm{e}+1$ & 128 & 16 & 18 & 13.7 & $2.1 \mathrm{e}+2$ \\
32 & 32 & 16 & 13.6 & $3.3 \mathrm{e}+2$ & 128 & 32 & 19 & 13.9 & $1.5 \mathrm{e}+3$ \\
\hline
\end{tabular}

\section{References}

1. Yves Achdou, Yvon Maday, and Olof B. Widlund. Iterative substructuring preconditioners for mortar element methods in two dimensions. SIAM J. Numer. Anal., 36:551-580, 1999.

2. Christine Bernardi, Yvon Maday, and Anthony Patera. A new non conforming approach to domain decomposition: The mortar element method. In H. Brezis and J.-L. Lions, editors, Collège de France Seminar. Pitman, 1994.

3. Maksymilian Dryja. An iterative substructuring method for elliptic mortar finite element problems with a new coarse space. East-West J. Numer. Math., 5(2):79-98, 1997.

4. Charbel Farhat, Michel Lesoinne, Patrick Le Tallec, Kendall Pierson, and Daniel Rixen. FETI-DP: A Dual-Primal FETI method - part I: A faster alternative to the two-level FETI method. Int. J. Numer. Meth. Eng., 50:1523-1544, 2001.

5. Charbel Farhat and François-Xavier Roux. A method of finite element tearing and interconnecting and its parallel solution algorithm. Int. J. Numer. Meth. Eng., 32:1205-1227, 1991.

6. Axel Klawonn and Olof B. Widlund. FETI and Neumann-Neumann iterative substructuring methods: Connections and new results. Comm. Pure Appl. Math., 54(1):57-90, 2001.

7. Jan Mandel. Balancing domain decomposition. Comm. Numer. Meth. Eng., 9:233-241, 1993.

8. Jan Mandel and Marian Brezina. Balancing domain decomposition for problems with large jumps in coefficients. Math. Comp., 65:1387-1401, 1996.

9. Jan Mandel and Clark R. Dohrmann. Convergence of a balancing domain decomposition by constraints and energy minimization. Numer. Lin. Alg. Appl., 10(7):639-659, 2003.

10. Jan Mandel, Clark R. Dohrmann, and Radek Tezaur. An algebraic theory for primal and dual substructuring methods by constraints. App. Num. Math., 2004. To appear.

11. Barry F. Smith, Petter Bjørstad, and William Gropp. Domain Decomposition: Parallel Multilevel Methods for Elliptic Partial Differential Equations. Cambridge University Press, 1996.

12. Dan Stefanica. A balancing algorithm for mortar finite elements. 2005. Preprint. 